Research Article

\title{
Combined Single-Source and Multi-source Capacitated Facility Location Problems with Data Envelopment Analysis
}

\author{
Ali Jamalian $\mathbb{D i}^{1}$ and Maziar Salahi $\mathbb{D D}^{2}$ \\ ${ }^{1}$ Department of Computer Science, Faculty of Mathematical Sciences, University of Guilan, Rasht 4199613776, Iran \\ ${ }^{2}$ Department of Applied Mathematics, Faculty of Mathematical Sciences and Center of Excellence for Mathematical Modeling, \\ Optimization and Combinatorial Computing (MMOCC), University of Guilan, Rasht 4199613776, Iran
}

Correspondence should be addressed to Ali Jamalian; a.jamalian.math@gmail.com

Received 27 August 2020; Revised 23 September 2020; Accepted 6 October 2020; Published 22 October 2020

Academic Editor: S. A. Edalatpanah

Copyright (C) 2020 Ali Jamalian and Maziar Salahi. This is an open access article distributed under the Creative Commons Attribution License, which permits unrestricted use, distribution, and reproduction in any medium, provided the original work is properly cited.

In this paper, we incorporate an efficiency criterion using data envelopment analysis into the single-source and multi-source capacitated facility location problems. We develop two bi-objective integer programs to find optimal and efficient location patterns, simultaneously. The proposed models for these capacitated facility location problems have fewer variables and constraints compared to existing models presented in the literature. We use the LP-metric procedure to solve the proposed models on two numerical examples. Results show that new models give better solutions, based on cost and efficiency criteria.

\section{Introduction}

Facility location problem (FLP) is concerned with finding optimal locations of facilities and how to allocate them to satisfy customers' demands such that the total fixed opening costs of facilities and the total transportation costs are minimized. It has applications in supply chain network design, telecommunication, public sector services, and distribution network design [1-4]. In location analysis, patterns with more output and service produced for a given amount of resources are more efficient and less wasteful. Attributes such as availability of infrastructures, resource usage, and receptiveness and perceptions of local population can influence performance of location pattern. Thus, considering a multi-objective program which includes different criteria makes sense. There are different methods to evaluate efficiency and performance, where, in this paper, we use data envelopment analysis (DEA)-based models. These models are quantitative and nonparametric mathematical programming-based approaches to evaluate efficiency. They can be used in combination with FLP models to produce integrated models that can be solved in one step in order to find best locations for facilities and efficient allocation pattern.
The DEA technique has extensive applications in different fields such as performance analysis [5], ranking companies or decision-making units $[5,6]$, efficient banking [7], industrial management [6, 7], and forecasting profitability in markets [8,9]. Moreover, applications of DEA models with the indeterminacy, impreciseness, vagueness, inconsistent, and incompleteness information are also widely studied $[10,11]$.

In recent works, FLP models are combined with DEA models to achieve best locations and maximum efficiency at the same time. Cook and Green [12] used DEA to select sites for facilities with a single resource restriction on operating budget. They proposed a mathematical programming model to find locations of sites for a set of retail outlets such that maximize the ratio of benefits to costs. Klimberg and Ratick [13] have utilized concept of DEA in order to formulate and find optimal and efficient facility location/allocation patterns. They used this concept for both uncapacitated and capacitated facility location problems. They proposed two bi-objective models for combined DEA and location-allocation models in which the total costs and average DEA efficiency objectives are optimized simultaneously. Karbasian and Dashti [14] used simultaneous DEA for four discrete, deterministic, uncapacitated, and static dispersion 
facility location problems. They proposed different multiobjective models to find optimal and efficient facility location patterns and maximize total demands of satisfied customers using the DEA method for $p$-dispersion problem, $p$-defense problem, and MaxMinSum and MaxSumSum dispersion and covering location problems in the presence of existing facilities. They also used a fuzzy goal programming method to solve their multi-objective models. Thomas et al. [15] used DEA in locating obnoxious facilities and presented two approaches in their study. In the first one, obtained optimum locations of facilities are considered as input of the DEA model. In the second one, a hierarchical process of DEA is used by a single-objective linear programming (LP) model that maximizes the efficiency of $p$ obnoxious facilities to be opened. In their study, the performance is considered in terms of proximity and DEA efficiency scores. Azadeh et al. [16] proposed an integrated hierarchical approach to select the most efficient and best-possible location for solar plants using the DEA approach. The optimum locations of solar power plants are ranked by DEA with respect to some output and input parameters. Also, principal component analysis and taxonomy methods are used to validate results of the model. Mitropoulos et al. [17] used combination of DEA approach to location planning of services and effective allocation of scarce resources such as equipment, funds, or workforce in the health sector. They proposed a framework to evaluate technical efficiency of existing healthcare centers and location analysis in order to maximize accessibility, utilization, and mean efficiency and select the appropriate number and locations of providers. Location analysis determines which centers will be upgraded and expanded and which ones will be closed. Moheb-Alizadeh et al. [18] studied incorporation of DEA and location-allocation models in a fuzzy environment. They used the multicriterion form of DEA and simultaneously considered both facility location and demand assignment problems in which the number of facilities to be located was not predetermined. The demand of each product for each customer, the amount of resources that each facility uses, and the output of each facility in each candidate location are assumed as a fuzzy number. Adabi and Omrani [19] studied considering efficiency in design of supply chain and proposed a bi-objective mixed integer linear programming (MILP) where one objective maximized system efficiency of the network and the other one minimized the total setup and transportation costs of the pattern. Mohaghar et al. [20] developed an integration of fuzzy VIKOR and assurance region-DEA for selection and ranking suppliers in a supply chain network. Georgantzinos and Giannikos [21] also considered the incorporation of efficiency in the context of the set covering, set partitioning problem, and set packing problem. Finally, recently Houng and Jeong [22] combined DEA and multi-objective optimization techniques for the efficient facility location-allocation decisions and patterns to help practitioners and decision-makers.

Among FLPs, two widely used and studied problems are single-source capacitated facility location problem (SSCFLP) and multi-source capacitated facility location problem (MSCFLP) $[2,3]$. In this paper, we combine SSCFLP and
MSCFLP models with the CCR model of DEA. The resulting models are integer and mixed integer multi-objective LPs, respectively. In MSCFLP case, our model has less variables and constraints and gives better solutions compared to [13]. In both models, efficiency is defined as weighted sum of the outputs. To solve the proposed multi-objective models, we use the LP-metric method.

The remainder of this paper is organized as follows. Section 2 introduces single-source and multi-source capacitated facility location problems. Section 3 presents DEA models which are used in this paper. The proposed combined models of DEA with SSCFLP and MSCFLP are given in Section 4. Section 5 presents the solution procedure for the proposed models. Finally, numerical examples are given in Section 6.

\section{Capacitated Facility Location Problems}

In what follows, we give MSCFLP and SSCFLP models. The MSCFLP can be formulated as the following MILP:

$$
\begin{gathered}
\min \sum_{h} \sum_{l} c_{h l} x_{h l}+\sum_{h} f_{h} y_{h}, \\
\text { s.t. } \sum_{h} x_{h l} \geq a_{l}, \quad \forall l, \\
\sum_{l} x_{h l} \leq b_{h} y_{h}, \quad \forall h, \\
y_{h} \in\{0,1\}, \quad \forall h, \\
x_{h l} \geq 0, \quad \forall l, \forall h,
\end{gathered}
$$

where $h=1, \ldots, H$ is the index of facility locations, $l=1, \ldots, L$ is the index of demand points, $c_{h l}$ is the cost of shipping one unit of demand from facility $h$ to demand point $l, a_{l}$ is the number of units of demand at $l, b_{h}$ is the capacity of facility $h, f_{h}$ is the fixed cost of opening facility $h$, and $x_{h l}$ is the fraction of demand at point $l$ satisfied from facility $h$ :

$$
y_{h}= \begin{cases}1, & \text { if facility } h \text { is opened, } \\ 0, & \text { o.w. }\end{cases}
$$

Objective function (1) calculates the total fixed opening and transportation costs. The transportation costs are calculated as the product of the per unit transportation costs and the amount shipped from facility $h$ to demand $l$. Constraints (2) ensure that every demand point is satisfied, and constraints (3) ensure that only open facilities can supply demand points considering their capacity. Finally, constraints (4) and (5) are binary and nonnegative constraints on variables, respectively.

In SSCFLP, customers are forced to be served only from a single facility which is applicable for real-world situations where multiple deliveries may increase the cost of maintaining and updating the inventory [23]. It can be formulated as the following ILP: 


$$
\begin{aligned}
& \min \sum_{h} \sum_{l} a_{l} c_{h l} x_{h l}+\sum_{h} f_{h} y_{h}, \\
& \text { s.t. } \sum_{h} x_{h l}=1, \quad \forall l, \\
& \sum_{l} a_{l} x_{h l} \leq b_{h} y_{h}, \quad \forall h, \\
& y_{h}, x_{h l} \in\{0,1\}, \quad \forall l, \forall h,
\end{aligned}
$$

where

$$
\begin{aligned}
& y_{h}= \begin{cases}1, & \text { if facility } h \text { is opened } \\
0, & \text { o.w }\end{cases} \\
& x_{h l}= \begin{cases}1, & \text { if facility } h \text { serves demand } l, \\
0, & \text { o.w. }\end{cases}
\end{aligned}
$$

Objective function (7) calculates the total fixed opening and transportation costs. The transportation costs are calculated as the product of the per unit transportation costs and the amount shipped from facility $h$ to demand $l$. Constraints (8) ensure that every demand point is satisfied, and constraints (9) ensure that only open facilities can supply demand points considering their capacity. Finally, constraints (10) are binary constraints on variables.

\section{Data Envelopment Analysis}

DEA is a nonparametric method based on mathematical programming for measuring relative efficiency of multiple homogeneous decision-making units (DMUs) with the same inputs and outputs. DEA is an effective tool for performance benchmarking when multiple performance measures exist and a priori information on the tradeoffs among these measures is completely available [6]. Farrell proposed a nonparametric approach for evaluating the efficiency of DMUs applying two inputs and one outputs, and later, Charnes et al. developed this approach for several inputs and outputs $[24,25]$. Evaluation of DMUs is done by the ratio of the weighted sum of outputs to the weighted sum of inputs which is relative efficiency of them. In this manner, DEA finds the weights such that the efficiency of each DMU is maximized rather than the other DMUs. The multiplier form of the CCR (CCRm) DEA model evaluating the efficiency of $o$-th DMU is as follows:

$$
\begin{aligned}
& \max \frac{\sum_{r} O_{r o} u_{r}}{\sum_{i} I_{i o} v_{i}}, \\
& \text { s.t. } \frac{\sum_{r} O_{r j} u_{r}}{\sum_{i} I_{i j} v_{i}} \leq 1, \quad \forall j, \\
& u_{r}, v_{i} \geq 0, \quad \forall i, \forall r,
\end{aligned}
$$

where $n$ is number of DMUs, $m$ is number of inputs, $s$ is number of outputs, $I_{i j}$ is the $i$-th input value for $j$-th DMU, $O_{r j}$ is the $r$-th output value for $j$-th DMU, $u_{r}$ is the weight values of the $r$-th output, and $v_{i}$ is the weight values of the $i$ th input. The objective function, which is the efficiency score of under evaluation DMU, cannot exceed unity, and DMUs are classified into two types based on their scores. The DMUs with an efficiency score 1 are called efficient, and others are called inefficient [5]. Charnes et al. transformed the CCRm to an LP as follows [25]:

$\max \sum_{r} O_{r o} u_{r}$

$$
\begin{aligned}
& \text { s.t. } \sum_{r} O_{r j} u_{r}-\sum_{i} I_{i j} v_{i} \leq 0, \quad \forall j, \\
& \sum_{i} I_{i o} v_{i}=1 \\
& u_{r}, v_{i} \geq \varepsilon, \quad \forall i, \quad \forall r
\end{aligned}
$$

where $\varepsilon$ is non-Archimedean infinitesimal value to prevent numerous zeros in input and output weights. The CCRm is always feasible [5], for example, if $\varepsilon=0$, then because $I_{i j}$ are positive, $v=\left(\left(1 / I_{1, o}\right), 0, \ldots, 0\right), u=(0, \ldots, 0)$ is a feasible solution. However, model (15-18) is not feasible for every value of $\varepsilon$. To find a suitable value of $\varepsilon$, an additional model should be solved which its feasible region is exactly the feasible region of CCRm, while its objective function is $\max \varepsilon$.

Klimberg and Ratick modified the standard model of CCRm and proposed a model to calculate DEA efficiency scores of all the DMUs in one LP as follows [13]:

$$
\begin{aligned}
& \max \sum_{j} \sum_{r} O_{r j} u_{r j}, \\
& \text { s.t. } \sum_{r} O_{r k} u_{r j}-\sum_{i} I_{i k} v_{i j} \leq 0 \quad \forall j, k, j \neq k, \\
& \sum_{i} I_{i j} v_{i j}=1, \quad \forall j, \\
& u_{r j}, v_{i j} \geq \varepsilon, \quad \forall i, \quad \forall r, \forall j,
\end{aligned}
$$

where $u_{r j}$ is the weight values of the $r$-th output in the $j$-th DMU and $v_{i j}$ is the weight values of the $i$-th input in the $j$-th DMU. Similar to model (15)-(18), models (19)-(22) are also feasible, for example, $v=\left[\begin{array}{cccc}1 / I_{1,1} & 1 / I_{1,2} & \cdots & 1 / I_{1, n} \\ 0 & 0 & \cdots & 0 \\ \vdots & \vdots & \vdots & \vdots \\ 0 & 0 & \cdots & 0\end{array}\right]$, $u=\left[\begin{array}{ccc}0 & \cdots & 0 \\ \vdots & \ddots & \vdots \\ 0 & \cdots & 0\end{array}\right]$ 


\section{Combined MSCFLP-DEA and SSCFLP- DEA Models}

In this section, DEA methodology has been used as a tool for incorporating concept of efficiency into MSCFLP and SSCFLP models as another objective to provide optimum location pattern with respect to the total transportation and fixed costs and also the performance of facilities at different potential sites. The SSCFLP is an important variant of CFLPs in practical situations that a combined model for which is proposed in this section. In order to incorporate DEA in CFLP models, the DEA efficiency scores of all DMUs should be calculated in one LP. We develop combined CFLP-DEA models using models (19)-(22) which maximize the sum of efficiencies.

4.1. MSCFLP-DEA Model. Incorporating the above DEA model in the MSCFLP results in the following bi-objective formulation which we call it MSCFLP-DEA:

$$
\begin{aligned}
& \min \sum_{h} \sum_{l} c_{h l} x_{h l}+\sum_{h} f_{h} y_{h}, \\
& \max \sum_{h} \sum_{l} \sum_{r} O_{r h l} u_{r h l}, \\
& \text { s.t. } \sum_{h} x_{h l} \geq a_{l}, \quad \forall l, \\
& \sum_{l} x_{h l} \leq b_{h} y_{h}, \quad \forall h, \\
& x_{h l} \leq M_{h l} z_{h l}, \quad \forall h, \forall l, \\
& z_{h l} \leq x_{h l}, \quad \forall h, \quad \forall l, \\
& \sum_{i} I_{i h l} v_{i h l}=z_{h l}, \quad \forall h, \forall l, \\
& y_{h}, z_{h l} \in\{0,1\}, \quad \forall l, \forall h, \quad \forall i, \quad \forall h, \\
& x_{h l} \geq 0, \quad \forall l, \quad \forall h, \forall l, \\
& \text { O }_{r h l} u_{r h l} \leq z_{h l}, \quad \forall h, \forall l, \forall r, \\
& \sum_{r} O_{r p q} u_{r h l}-\sum_{i} I_{i p q} v_{i h l} \leq 0, \quad \forall h, l, p, q, \quad p \neq h, q \neq l,
\end{aligned}
$$

where $M_{h l}=\min \left\{a_{l}, b_{h}\right\}, \varepsilon$ is a very small positive number, $I_{i h l}$ is the $i$-th input value for the link of facility $h$ and demand point $l, O_{r h l}$ is the $r$-th output value for the link of facility $h$ and demand point $l, v_{i h l}$ is the weight value of $i$-th input in the link of facility $h$ and demand point $l$, and $u_{r h l}$ is the weight value of $i$-th output in the link of facility $h$ and demand point $l$. First objective function (23) and constraints (25) and (26) are related to the MSCFLP model in (1)-(5). Moreover, we need extra binary variables $z_{h l}$ to show allocation status between facilities and demand points which is defined as follows:

$$
z_{h l}= \begin{cases}1, & \text { if } x_{h l}>0 \\ 0, & \text { o.w. }\end{cases}
$$

Constraints (27) and (28) are the reformulated form of above-fixed charge constraint which is added to the model. Objective function (25) and constraints (29)-(33) correspond to the DEA model in (19)-(22). Constraints (34) and (35) show the types of variables. Since both models (1)-(5) and (19)-(22) are feasible, and MSCFLP-DEA is a direct combination of them, then it is also feasible. A feasible solution is as follows: if $x_{h l}=0$, then let $v_{:, h, l}=(0, \ldots, 0), u_{:, h, l}=(0, \ldots$, $0), y_{h}=0$, and $z_{h l}=0$, and if $x_{h l}>0$, then $z_{h l}=1$ and let $v_{:, h, l}=$ $\left(1 / I_{1, h, l}, 0, \ldots, 0\right), u_{:, h, l}=(0, \ldots, 0)$, and $y_{h}=1$.

4.2. SSCFLP-DEA Model. Incorporating the DEA model in the SSCFLP results in the following bi-objective formulation which we call SSCFLP-DEA:

$$
\begin{aligned}
& \min \sum_{h} \sum_{l} a_{l} c_{h l} x_{h l}+\sum_{h} f_{h} y_{h}, \\
& \max \sum_{h} \sum_{l} \sum_{r} O_{r h l} u_{r h l}, \\
& \text { s.t. } \sum_{h} x_{h l}=1, \quad \forall l, \\
& \sum_{l} a_{l} x_{h l} \leq b_{h} y_{h}, \quad \forall h, \\
& \sum_{i} I_{i h l} v_{i h l}=x_{h l}, \quad \forall h, \forall l, \\
& O_{r h l} u_{r h l} \leq x_{h l}, \quad \forall h, \forall l, \forall r, \\
& \sum_{r} O_{r p q} u_{r h l}-\sum_{i} I_{i p q} v_{i h l} \leq 0, \quad \forall h, l, p, q, \quad p \neq h, q \neq l, \\
& y_{h}, x_{h l} \in\{0,1\}, \quad \forall l, \forall h . \\
& u_{r h l} \geq \varepsilon x_{h l}, \quad \forall r, \quad \forall h, \forall l,
\end{aligned}
$$

First objective function (37) and constraints (39) and (40) are related to the SSCFLP similar to the model in (7)-(10). Objective function (38) and constraints (41)-(45) are correspond to the DEA model in (19)-(22). Additionally, if facility $h$ does not serve the demand point $l, x_{h l}=0$, 
TABLE 1: Results of solving the MSCFLP-DEA model for example 1.

\begin{tabular}{|c|c|c|c|c|c|c|c|}
\hline$\omega$ & 0 & 0.5 & 0.6 & 0.7 & 0.8 & 0.9 & 1 \\
\hline \multicolumn{8}{|l|}{ Objectives } \\
\hline Fixed costs & 2620 & 2620 & 2320 & 1950 & 1510 & 1510 & 1010 \\
\hline Transportation costs & 17789 & 11057 & 10795 & 10677 & 10165 & 10165 & 10497 \\
\hline Total costs $\left(Z_{1}^{*}\right)$ & 20409 & 13677 & 13115 & 12627 & 11675 & 11675 & 11507 \\
\hline Sum of efficiency scores $\left(Z_{2}^{*}\right)$ & 83.5998 & 83.5998 & 78.0917 & 70.2095 & 45.8466 & 45.8466 & 6.216 \\
\hline \multicolumn{8}{|l|}{$\overline{\text { DMUs }}$} \\
\hline No. of open facilities & 7 & 7 & 6 & 5 & 4 & 4 & 3 \\
\hline No. of links & 105 & 105 & 90 & 75 & 60 & 60 & 45 \\
\hline Average score of links & 0.7961 & 0.7961 & 0.8676 & 0.9361 & 0.7641 & 0.7641 & 0.1381 \\
\hline Minimum score of links & 0.1493 & 0.1493 & 0.1493 & 0.1493 & 0.1493 & 0.1493 & 0.043 \\
\hline
\end{tabular}

constraints (41) and (42) force input and output weights to be zero. So, the efficiency of a facility-demand link is equal to 0 if it is not used. Constraints (46) show the types of variables. Similar to the previous model, here also one can easily show the feasibility of SSCFLP-DEA.

\section{Solution Procedure}

Since MSCFLP-DEA and SSCFLP-DEA models are bi-objective with inconsistent objective functions, we use the LPmetric method [26] which is one of the famous and useful methods for solving multi-objective problems with conflicting objectives. This method considers each objective function separately and reformulate a single objective to minimize the sum of the normalized differences between the objectives and their optimal values. The two objective functions are denoted by $Z_{1}$ and $Z_{2}$. Based on the LP-metric method, MSCFLP-DEA and SSCFLP-DEA should be solved for each one of $Z_{1}$ and $Z_{2}$ separately. Objective function of the LP-metrics can be formulated as follows:

$$
\min Z_{3}=\omega \frac{Z_{1}-Z_{1}^{*}}{Z_{1}^{*}}+(1-\omega) \frac{Z_{2}-Z_{2}^{*}}{Z_{2}^{*}},
$$

where $0 \leq \omega \leq 1$ is the weight which indicates relative importance of the two objective functions and $Z_{1}^{*}$ and $Z_{2}^{*}$ are optimum values of $Z_{1}$ and $Z_{2}$, respectively. Using LP-metric objective function $Z_{3}$, we have single-objective models which can be solved by efficient solvers such as CPLEX and Gurobi [27, 28].

\section{Numerical Examples}

In this section, we demonstrate the MSCFLP-DEA and SSCFLP-DEA models on two examples. In both examples, each MSCFLP-DEA and SSCFLP-DEA models are optimally solved three times for objective functions $Z_{1}, Z_{2}$, and $Z_{3}$. Objective function $Z_{1}$ minimizes the total fixed and transportation costs. The function $Z_{2}$ maximizes the sum of efficiencies of DMUs. The third objective function $Z_{3}$ is the LP-metric objective function which uses best values of $Z_{1}$ and $Z_{2}$ to make a tradeoff between costs and efficiency scores of the facility-demand links. We solve the models using IBM ILOG CPLEX 12.5 on a CORE2Duo CPU of 2 GHZ and 2GB of RAM.
6.1. Example 1. This example is taken from [13] and includes seven facilities $\left(F_{1}-F_{7}\right)$, fifteen demand points $\left(D_{1}-D_{15}\right)$, four inputs $\left(I_{1}-I_{4}\right)$, and three outputs $\left(O_{1}-O_{3}\right)$. Results are given in Tables 1 and 2 for the MSCFLP-DEA and the SSCFLP-DEA, respectively.

6.2. Example 2. This example is taken from [18] and includes five facilities $\left(F_{1}-F_{5}\right)$, eight demand points $\left(D_{1}-D_{8}\right)$, three inputs $\left(I_{1}-I_{3}\right)$, and two outputs $\left(O_{1}-O_{2}\right)$. Results are given in Tables 3 and 4 for the MSCFLP-DEA and the SSCFLP-DEA, respectively.

Figure 1 illustrates the tradeoff between the total costs and the sum of efficiency scores of DMUs for $w=1$, $0.9,0.8,0.7,0.6,0.5$, and 0 , respectively. This curve is an efficient frontier, and the decision-maker can select a suitable value for $w$. As it is shown, sum of efficiencies of facility-demand links increases by increasing the total costs. It is worthy that the slope of increasing efficiency is higher at the first and then it decreases. In example 1, the average of efficiency scores $\% 84$ increases with only $\% 12$ increase in the costs. So, it means that we can find efficient patterns with a little more costs which is valuable. Figure 2 and 3 show the effect of weight of LP-metric objective function on the total costs and efficiency scores, respectively. Figure 4 shows the relative variation of efficiency scores by relative variation of total costs. As we see in Figure 4, \%1.44 increase in the costs of locations pattern leads to $\% 81.93$ increase in the efficiency of pattern. The maximum efficiency is achieved by about $\% 8.87$ increase in the costs.

Results of solving the SSCFLP-DEA model in example 1 are shown in Table 2 . Figure 5 represents the tradeoff between the total costs and the sum of efficiency scores for $w=1,0.8,0.6,0.5,0.4,0.2$, and 0 , respectively. As it is shown, sum of efficiencies of facility-demand links increases by increasing the total cost. The slope of increasing efficiency decreases by costs. The average of efficiency scores $\% 71.31$ increases by changing some assignments and without any additional costs. Figure 6 and 7 show the effect of weight of LP-metric objective function on the total costs and efficiency scores, respectively. Total costs decrease by increasing the weight, and sum of efficiencies increases by decreasing it. Figure 8 shows that relative variation of efficiency scores by relative variation of total cost. \% 11.38 increase in the costs of 
TABLE 2: Results of solving the SSCFLP-DEA model for example 1.

\begin{tabular}{|c|c|c|c|c|c|c|c|}
\hline$\omega$ & 0 & 0.2 & 0.4 & 0.5 & 0.6 & 0.8 & 1 \\
\hline \multicolumn{8}{|l|}{ Objectives } \\
\hline Fixed costs & 2620 & 1370 & 1620 & 1620 & 1950 & 1880 & 1880 \\
\hline Transportation costs & 26927 & 13768 & 12408 & 11596 & 8897 & 7733 & 7733 \\
\hline Total costs $\left(Z_{1}^{*}\right)$ & 29547 & 15138 & 14028 & 13216 & 10847 & 9613 & 9613 \\
\hline Sum of efficiency scores $\left(Z_{2}^{*}\right)$ & 29.3766 & 26.4868 & 25.2418 & 23.4283 & 14.3910 & 8.0088 & 2.2980 \\
\hline \multicolumn{8}{|l|}{ DMUs } \\
\hline No. of open facilities & 7 & 5 & 5 & 4 & 4 & 3 & 3 \\
\hline Average score of links & 1.958 & 1.7657 & 1.6827 & 1.56188 & 1.5618 & 0.5339 & 0.1532 \\
\hline Minimum score of links & 1.2312 & 0.5644 & 0.1888 & 0.1888 & 0.1888 & 0.1888 & 0.064 \\
\hline
\end{tabular}

TABLE 3: Results of solving the MSCFLP-DEA model for example 2.

\begin{tabular}{|c|c|c|c|c|c|c|c|}
\hline$\omega$ & 0 & 0.2 & 0.4 & 0.5 & 0.6 & 0.8 & 1 \\
\hline \multicolumn{8}{|l|}{ Objectives } \\
\hline Fixed costs & 1251 & 1251 & 1251 & 1251 & 753 & 500 & 500 \\
\hline Transportation costs & 9392 & 6257 & 6257 & 6257 & 5720 & 5449 & 5449 \\
\hline Total costs $\left(Z_{1}^{*}\right)$ & 10643 & 7508 & 7508 & 7508 & 6473 & 5949 & 5949 \\
\hline Sum of efficiency scores $\left(Z_{2}^{*}\right)$ & 28.1392 & 28.1392 & 28.1392 & 28.1392 & 22.6252 & 18.8038 & 1.961 \\
\hline \multicolumn{8}{|l|}{ DMUs } \\
\hline No. of open facilities & 5 & 5 & 5 & 5 & 3 & 2 & 2 \\
\hline No. of links & 40 & 40 & 40 & 40 & 24 & 16 & 16 \\
\hline Average score of links & 0.7034 & 0.7034 & 0.7034 & 0.7034 & 0.9427 & 1.1752 & 0.1225 \\
\hline Minimum score of links & 0.2156 & 0.2156 & 0.2156 & 0.2156 & 0.3220 & 0.3220 & 0.046 \\
\hline
\end{tabular}

TABLE 4: Results of solving the SSCFLP-DEA model for example 2.

\begin{tabular}{|c|c|c|c|c|c|c|c|}
\hline$\omega$ & 0 & 0.2 & 0.4 & 0.5 & 0.6 & 0.8 & 1 \\
\hline \multicolumn{8}{|l|}{ Objectives } \\
\hline Fixed costs & 1251 & 500 & 500 & 500 & 500 & 500 & 500 \\
\hline Transportation costs & 9018 & 6315 & 5755 & 5755 & 5755 & 5228 & 5228 \\
\hline Total costs $\left(Z_{1}^{*}\right)$ & 10269 & 6815 & 6255 & 6255 & 6255 & 5728 & 5728 \\
\hline Sum of efficiency scores $\left(Z_{2}^{*}\right)$ & 14.6151 & 13.2075 & 12.2591 & 12.2591 & 12.2591 & 9.5926 & 0.959 \\
\hline \multicolumn{8}{|l|}{ DMUs } \\
\hline No. of open facilities & 5 & 2 & 2 & 2 & 2 & 2 & 2 \\
\hline Average score of links & 1.8268 & 1.6509 & 1.5323 & 1.5323 & 1.5323 & 1.1990 & 0.1198 \\
\hline Minimum score of links & 1.2467 & 0.5923 & 0.5012 & 0.5012 & 0.5012 & 0.5012 & 0.0460 \\
\hline
\end{tabular}

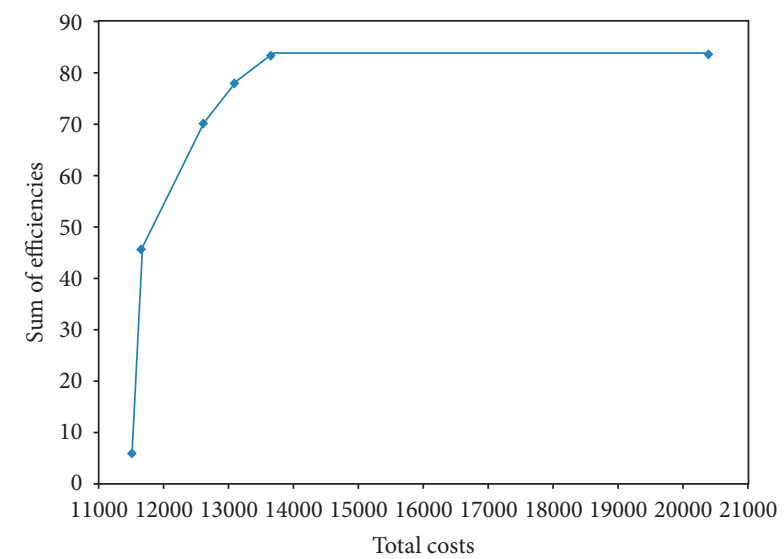

Figure 1: Total costs vs. efficiency scores.

locations pattern leads to $\% 90.19$ increase in efficiencies. The maximum efficiency is achieved by about $\% 67.47$ increase in the costs.

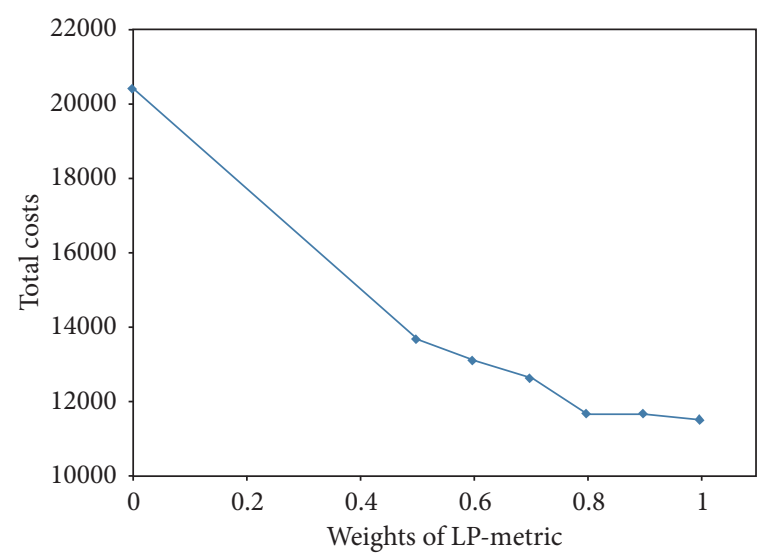

Figure 2: Weight vs. total costs.

Similar results hold for example 2. Sum of efficiencies of the links increases by increasing the total costs, and an efficient pattern can be achieved by a little additional cost. 


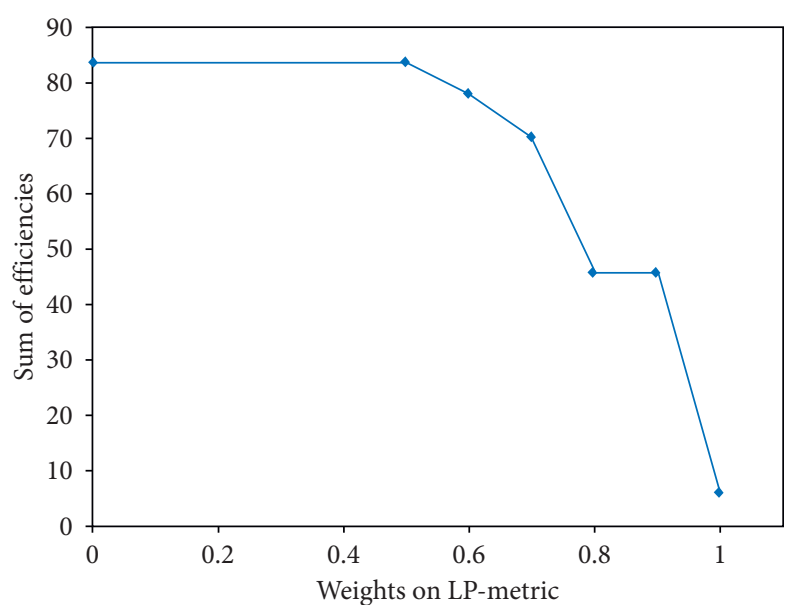

FIgURE 3: Weight vs. efficiency scores.

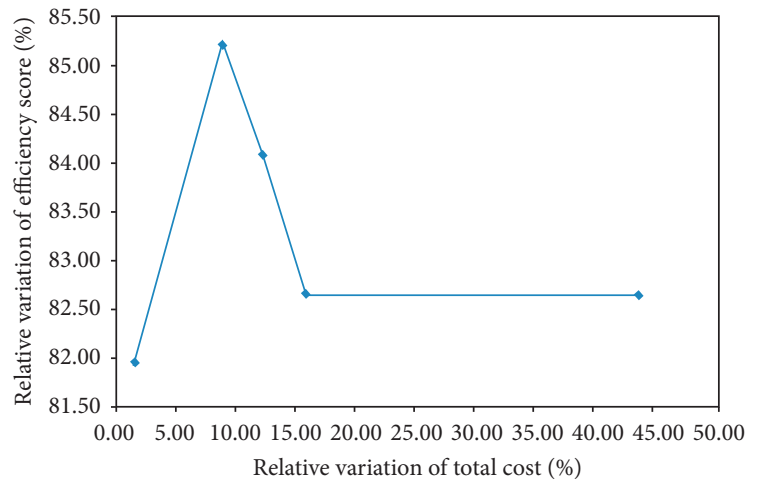

Figure 4: Relative variation of efficiency scores vs. total costs.

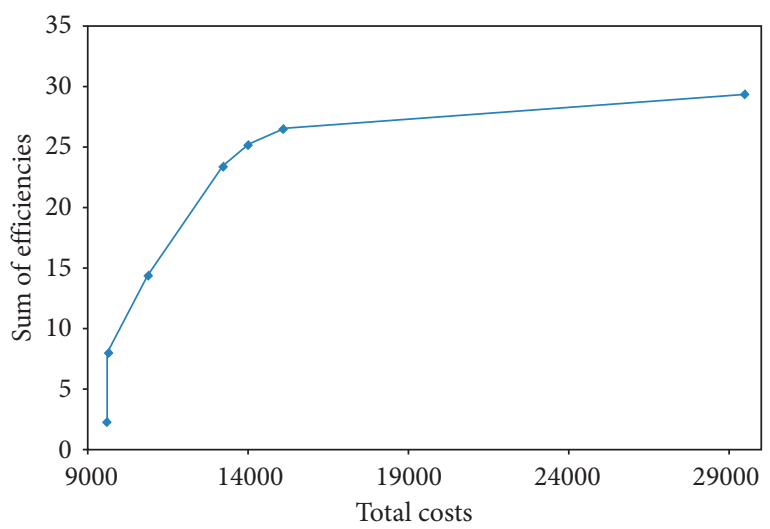

Figure 5: Total costs vs. efficiency scores.

The results of examples 1 and 2 confirm that the optimum solutions of the MSCFLP and the SSCFLP do not necessarily have efficient location-allocation pattern. In other words, the solutions of these problems under the cost criterion are different from the ones under efficiency criterion. So, to increase efficiency of allocations in the MSCFLP and the SSCFLP, more fixed cost or transportation costs are needed. It means that the total cost and the efficiency of the locationallocation pattern are conflicting objectives. The results of

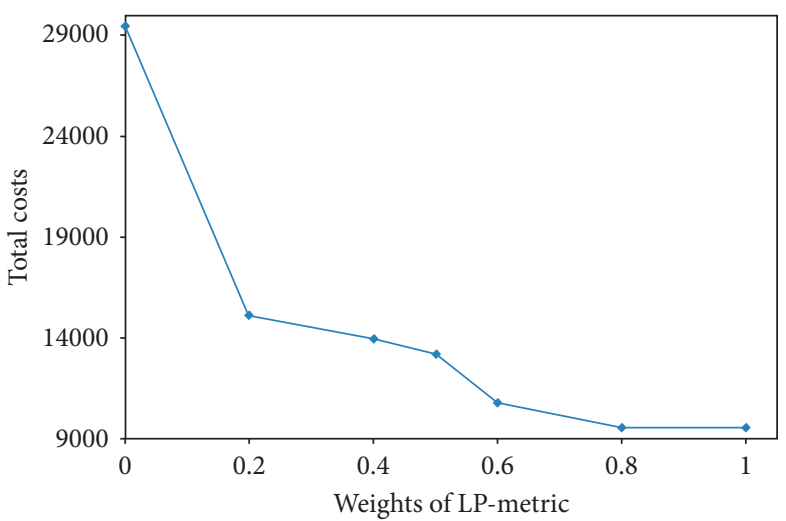

Figure 6: Weight vs. total costs.

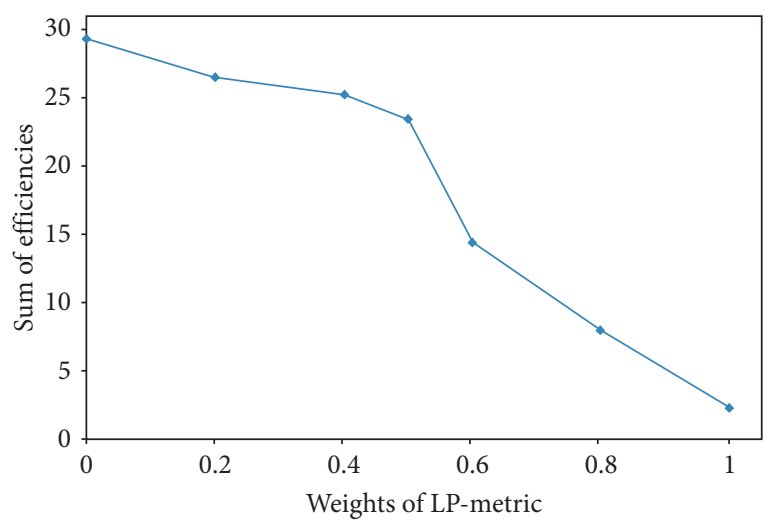

Figure 7: Weight vs. efficiency scores.

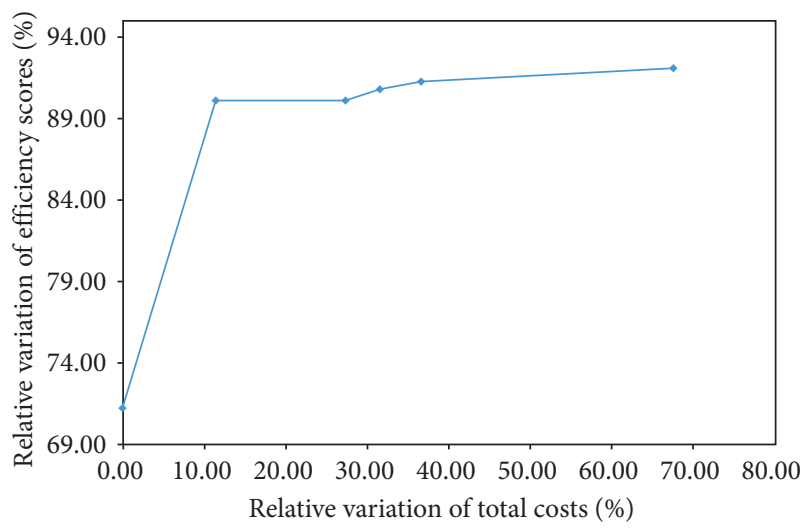

FIGURE 8: Relative variation of efficiency scores vs. total costs.

these examples show that, in a location-allocation pattern which has minimum cost, achieving high level of efficiency is cheap.

\section{Conclusions}

In this paper, we have incorporated the concept of the efficiency into two kinds of capacitated facility location problems, SSCFLP and MSCFLP, using DEA models. We have proposed two bi-objective integer programs for SSCFLP-DEA and MSCFLP-DEA models using the CCR 
model of DEA for performance evaluation and measuring efficiency. The developed model for MSCFLP-DEA has less variables and constraints compared with the one proposed by Klimberg and Ratick in [13]. In both SSCFLP-DEA and MSCFLP-DEA models, the number of facilities to be located is not predetermined and the models should specify it. Our models can determine location pattern including location of facilities and assignment of customers to them and also consider efficiency scores simultaneously. We used an LPmetric procedure to solve the bi-objective models, and computational results on two numerical examples show that these models are more reliable and efficient. Results of numerical examples show that the high level of efficiency can be achieved by only little increase in the costs. Due to the uncertainty of data in real-world applications, studying this problem under uncertainty can be considered as a future research direction.

\section{Data Availability}

The data supporting examples of this manuscript are from previously reported studies and datasets, which have been cited in the manuscript. The data are available in following references: [R. K. Klimberg and S. J. Ratick, Modeling data envelopment analysis (DEA) efficient location/allocation decision. Computer \& Operation Research, vol. 35, pp. 457-474, 2008.] [M. Moheb-Alizadeh, S. M. Rasouli, and R. Tavakkoli-Moghaddam, "The use of multi-criteria data envelopment analysis (MCDEA) for location-allocation problems in a fuzzy environment", Expert Systems with Applications, vol. 38, pp. 5687-5695, 2011].

\section{Conflicts of Interest}

The authors declare that there are no conflicts of interest regarding the publication of this paper.

\section{References}

[1] M. S. Daskin, Network and Discrete Location, Models, Algorithms, and Applications, John Wiley \& Sons, Inc, New York, NY, USA, 1995.

[2] Z. Drezner and H. W. Hamacher, Facility Location: Theory and Algorithms, Springer, Berlin, Germany, 2001.

[3] R. Z. Farahani and M. Hekmatfar, Facility Location: Concepts, Models, Algorithms and Case Studies, Physica-Verlag HD, Heidelberg, Germany, 2009.

[4] H. A. Eiselt and V. Marianov, Foundations of Location Analysis, Springer, New York, NY, USA, 2011.

[5] W. W. Cooper, L. M. Seiford, and K. Tone, Data Envelopment Analysis: A Comprehensive Text with Models, Applications, References and DEA-Solver Software, Springer, New York, NY, USA, 2nd edition, 2007.

[6] J. Zhu, Quantitative Models for Performance Evaluation and Benchmarking: Data Envelopment Analysis with Spreadsheets and DEA Excel Solver, Kluwer Academic Publishers, Boston, MA, USA, 2003.

[7] A. Emrouznejad, J. Jablonský, R. Banker, and M. Toloo, Recent Applications of Data Envelopment Analysis, Aston Business School, Birmingham, UK, 2017.
[8] Z. Dai, X. Dong, J. Kang, and L. Hong, "Forecasting stock market returns: new technical indicators and two-step economic constraint method," The North American Journal of Economics and Finance, vol. 53, Article ID 101216, 2020.

[9] Z. Dai, H. Zhu, and J. Kang, "New technical indicators and stock returns predictability," International Review of Economics \& Finance, vol. 71, pp. 127-142, 2021, p.

[10] M. R. Soltani, S. A. Edalatpanah, F. M. Sobhani, and S. E. Najafi, "A novel two-stage DEA model in fuzzy environment: application to industrial workshops performance measurement," International Journal of Computational Intelligence Systems, vol. 13, no. 1, pp. 1134-1152, 2020.

[11] S. A. Edalatpanah, "Data envelopment analysis based on triangular neutrosophic numbers," CAAI Transactions on Intelligence Technology, vol. 5, no. 2, pp. 94-98, 2020, p.

[12] W. D. Cook and R. H. Green, "Selecting sites for new facilities using data envelopment analysis," Journal of Productivity Analysis, vol. 19, no. 1, pp. 77-91, 2003.

[13] R. K. Klimberg and S. J. Ratick, "Modeling data envelopment analysis (DEA) efficient location/allocation decisions," Computers \& Operations Research, vol. 35, no. 2, pp. 457-474, 2008.

[14] M. Karbasian and M. Dashti, "Designing four multi-objective models for dispersion facilities location problems considering data envelopment analysis and maximum covering," International Journal of Management Science and Engineering Management, vol. 6, no. 4, pp. 298-306, 2011.

[15] P. Thomas, Y. Chan, L. Lehmkuhl, and W. Nixon, "Obnoxious-facility location and data-envelopment analysis: a combined distance-based formulation," European Journal of Operational Research, vol. 141, no. 3, pp. 495-514, 2002.

[16] A. Azadeh, S. F. Ghaderi, and A. Maghsoudi, "Location optimization of solar plants by an integrated hierarchical DEA PCA approach," Energy Policy, vol. 36, no. 10, pp. 3993-4004, 2008.

[17] P. Mitropoulos, I. Mitropoulos, and I. Giannikos, “Combining DEA with location analysis for the effective consolidation of services in the health sector," Computers \& Operations Research, vol. 40, no. 9, pp. 2241-2250, 2013.

[18] H. Moheb-Alizadeh, S. M. Rasouli, and R. Tavakkoli-Moghaddam, "The use of multi-criteria data envelopment analysis (MCDEA) for location-allocation problems in a fuzzy environment," Expert Systems with Applications, vol. 38, no. 5, pp. 5687-5695, 2011.

[19] F. Adabi and H. Omrani, "Designing a supply chain management based on distributers' efficiency measurement," Uncertain Supply Chain Management, vol. 3, pp. 87-96, 2015.

[20] A. Mohaghar, M.R. Fathi, and A.H. Jafarzadeh, "A supplier selection method using AR-DEA and fuzzy vikor," International Journal of Industrial Engineering: Theory, Applications and Practice, vol. 20, no. 5-6, pp. 372-386, 2013.

[21] S. K. Georgantzinos and I. Giannikos, "A modeling framework for incorporating DEA efficiency into set covering, packing, and partitioning formulations," International Transactions in Operational Research, vol. 26, no. 6, pp. 2387-2409, 2019.

[22] J.-D. Hong and K.-Y. Jeong, "Combining data envelopment analysis and multi-objective model for the efficient facility location-allocation decision," Journal of Industrial Engineering International, vol. 15, no. 2, pp. 315-331, 2019.

[23] R. Sridharan, "The capacitated plant location problem," European Journal of Operational Research, vol. 87, no. 2, pp. 203-213, 1995. 
[24] M. J. Farrell, "The measurement of productive efficiency," Journal of the Royal Statistical Society. Series A (General), vol. 120, no. 3, pp. 253-281, 1957.

[25] A. Charnes, W. W. Cooper, and E. Rhodes, "Measuring the efficiency of decision making units," European Journal of Operational Research, vol. 2, no. 6, pp. 429-444, 1978.

[26] K. Deb, Multi-Objective Optimization Using Evolutionary Algorithms, John Wiley \& Sons, Inc, New York, NY, USA, 2001.

[27] "IBM ILOG CPLEX optimisation studio reference manual," 2020, https://www.ibm.com/products/ilog-cplex-optimizationstudio.

[28] "Gurobi optimizer reference manual," 2020, https://www. gurobi.com. 